\title{
On the correctness of mathematical models of time-of-flight cathodoluminescence of direct-gap semiconductors
}

\author{
Mikhail A. Stepovich ${ }^{1, *}$, Dmitry $V$. Turtin ${ }^{2}$, Elena $V$. Seregina ${ }^{3}$, \\ and Veronika $V$. Kalmanovich ${ }^{1}$ \\ ${ }^{1}$ Tsiolkovsky Kaluga State University, Phys. and Math. Department, 26 Stepan Razin Street, \\ 248023 Kaluga, Russia \\ ${ }^{2}$ Plekhanov Russian University of Economics, Ivanovo Branch, Department of Humanities and \\ Natural Sciences, 53 Dzerzhinsky Street, 153025 Ivanovo, Russia \\ ${ }^{3}$ Bauman Moscow State Technical University (National Research University), Kaluga Branch, \\ Department of Automatic Control Systems and Electrical Engineering, 2 Bazhenov Street, \\ 248000 Kaluga, Russia
}

\begin{abstract}
Two-dimensional and three-dimensional mathematical models of diffusion and cathodoluminescence of excitons in single-crystal gallium nitride excited by a pulsating sharply focused electron beam in a homogeneous semiconductor material are compared. The correctness of these models has been carried out, estimates have been obtained to evaluate the effect of errors in the initial data on the distribution of the diffusing excitons and the cathodoluminescence intensity.
\end{abstract}

\section{Introduction}

Registration of informative signals excited in a semiconductor target and comparison of experimental data with a mathematical model of this phenomenon make it possible to identify semiconductor parameters that are very difficult or even impossible to determine by other methods. The need for the study is also due to the insufficient knowledge of the physical phenomenon under consideration: there are only a few publications devoted to studies of the correctness of mathematical models used in electron beam technologies [1-4]. It can also be said that previously the task of modelling diffusion (and subsequent radiative recombination with the release of cathodoluminescent (CL) radiation from a semiconductor) for the process under consideration was to a certain extent semiquantitative. It was solved only when using the model of energy losses by primary lowenergy electrons in the target in the form of a two-dimensional normal Gaussian distribution, which is a rather rough approximation that describes the available experimental data on energy losses in a condensed matter only qualitatively [5, 6].

A mathematically correct study of mathematical models of physical phenomena arising from the interaction of electron beams with semiconductor objects and described by differential equations of heat and mass transfer with partial derivatives has practically not

* Corresponding author: m.stepovich@rambler.ru 
been carried out before [1-4]. We note that physical processes (in particular, diffusion and subsequent $\mathrm{CL}$ ) are reduced to mathematical models that are described by initial or boundary value problems for partial differential equations, therefore, in the study it is necessary to solve a number of the following problems [7]: 1) establishing the existence of a solution; 2) proof of the uniqueness of the solution; 3) substantiation of the continuous dependence of the solution on the problem data (initial and boundary data, free term, equation coefficients). Tasks that satisfy the requirements 1)-3) are called correctly posed. The study of this issue is very relevant, since the initial data and parameters of the problem have a measurement error, and with minor changes in the initial data, the solutions to the problem can vary dramatically [7]. The problem of the correctness of such models is rarely studied, due to great difficulties of a logical and technical nature, which also makes such studies very important. In particular, the solution to the problems of identifying the electrophysical parameters of semiconductors is largely determined by the correctness of the used mathematical models, which is the subject of consideration in this paper as applied to the diffusion of nonequilibrium minority charge carriers (MCC) or excitons generated by the electron beam and their subsequent radiative recombination.

\section{Time-of-flight cathodoluminescence}

For semiconductor optoelectronic materials, one of the most informative is CL radiation, the intensity $I$ of which at a fixed wavelength at time $t$ is proportional to the concentration of nonequilibrium MCC (or excitons) $c(M, t)$ generated by the electron beam in the semiconductor:

$$
I(t)=\iiint_{V} c(M, t) \exp (-\alpha z) d V
$$

Here $M$ is the coordinate of an arbitrary point of the target, in a Cartesian rectangular coordinate system, while the axes OX and OY lie on the flat surface of the semiconductor, and the axis $\mathrm{OZ}$ is directed deep into the target; $\alpha$ is absorption coefficient of CL radiation.

In practice, when registering a CL signal, a pulsed electron beam is usually used to increase the signal-to-noise ratio. In this case, for the problems of CL diagnostics of semiconductors, the most interesting is the use of a sharply focused electron beam, an electron probe. Such an experimental design is also used in time-of-flight CL measurements, the essence of which is as follows. A mask impervious to CL radiation is applied to the surface of the investigated semiconductor - see Fig. 1.

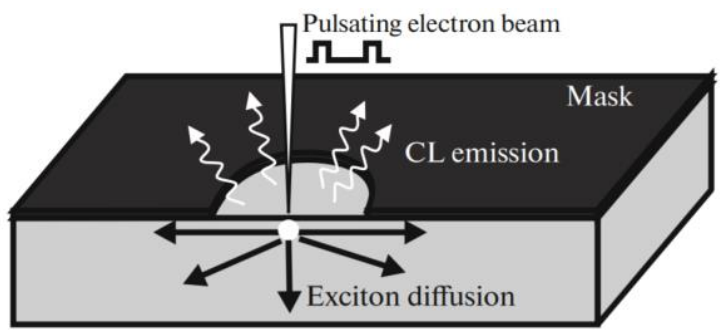

Fig. 1. The scheme of the simulated experiment [8-11].

The mask has a round hole of known radius. CL radiation is excited in the center of the hole using a pulsating electron beam and the radiation arising in the semiconductor is recorded in the spectral region characteristic of the recombination of generated particles, for example, free excitons in gallium nitride, a promising material in optoelectronics and microwave technology. 
After the equilibrium between the generation and recombination processes is established in the sample, the excitation ceases: the electron beam is deflected by the blanking system onto the mask, does not enter the semiconductor, and CL radiation is not excited. The character of the subsequent decrease in the CL intensity depends in the general case only on the known hole radius, exciton lifetime (in $\mathrm{GaN}$ ), which can be obtained from the CL measurements of the semiconductor under study before applying the mask, and the diffusion coefficient characterizing the motion of excitons. This can allow, in the presence of a mathematical model describing a decrease in the CL intensity, based on the analysis of experimental data, to obtain estimates of the exciton diffusion coefficient in the materials under study, in the general case, by solving the corresponding inverse problem. In mathematical modeling of lateral transport and radiative recombination of MCC, this problem is considered as two-dimensional - the use of such a model is justified at low electron probe energies $[1,2,4,10,11]$. This approach was also used in the present work; in this case, the energy losses by the electron probe in the target are described both using the Gaussian function [1,3] and using a mathematical model that takes into account the peculiarities of energy losses in the condensed matter absorbed in the target and backscattered electrons $[5,6]$.

\section{Mathematical model}

In the Cartesian coordinate system, the unsteady equation of diffusion of the nonequilibrium MCC, which describes the change in carrier concentration upon switching off the electron beam, has the form

$$
\frac{\partial c(M, t)}{\partial t}=D \Delta c(M, t)-\frac{c(M, t)}{\tau}
$$

with the initial condition $c(M, 0)=n(M)$, where $n(M)$ is the solution of the stationary differential equation that describes the diffusion of the MCC in a quasi-equilibrium state, which is realized under stationary conditions of irradiation of a semiconductor target:

$$
\Delta n(M)-\frac{n(M)}{\lambda^{2}}=-\rho(M)
$$

Here, $\lambda=\sqrt{D \tau}$ is the diffusion length, and $\rho(M)$ is the concentration of the MCC or excitons, determined by the energy loss density of the primary electron beam.

In the general case, the energy loss by an electron probe in a conducting target per unit time is given by the expression $[5,6]$ :

$$
\begin{gathered}
\rho^{*}(x, y, z)=\frac{1.085(1-\eta) P_{0}}{\pi^{3 / 2} a_{1}^{2} z_{m s}\left(1-\eta+\eta z_{s s} / z_{m s}\right)}\left\{\exp \left\{-\left[\frac{x^{2}+y^{2}}{a_{1}^{2}}+\left(\frac{z-z_{m s}}{z_{m s}}\right)^{2}\right]\right\}+\right. \\
\left.+\frac{\eta a_{1}^{2}}{(1-\eta) a_{2}^{2}} \exp \left\{-\left[\frac{x^{2}+y^{2}}{a_{2}^{2}}+\left(\frac{z-z_{s s}}{z_{s s}}\right)^{2}\right]\right\}\right\} .
\end{gathered}
$$

Here the origin coincides with the point of incidence of the electron beam on the sample; $P_{0}$ is the primary beam power; $z_{m s}$ is the depth of maximum energy loss with primary electrons, that have experienced small-angle scattering and absorbed in the target, and $z_{s s}$ 
is the depth of the maximum energy loss with backscattered electrons, $z_{\mathrm{ss}}=Z^{-1 / 3} z_{\mathrm{ms}}, Z$ is the effective target material number; $\eta$ is backscattering coefficient of electrons. Parameters $a_{1}$ and $a_{2}$ are determined from $a_{1}^{2}=z_{m s}^{2}+0.72 d_{b}^{2}$ and $a_{2}^{2}=0.25 z_{s s}^{2}+0.72 d_{b}^{2}$, respectively, where $d_{b}$ is diameter of the probe.

For probe electrons with energy $E_{0}[\mathrm{keV}]$ incident perpendicular to a target surface with density $\rho_{0}\left[\mathrm{~g} / \mathrm{cm}^{3}\right], z_{m s}$ can be expressed in terms of total beam electron path $R^{*}$ in a solid (see diffusion model [12]):

$$
z_{m s}[\mu \mathrm{m}]=\frac{R^{*}}{2}\left[1-\left(\frac{C \gamma}{1+\gamma}\right)^{2}\right], R^{*}[\mu \mathrm{m}]=\frac{2.76 \cdot 10^{-2} A E_{0}^{5 / 3}}{\rho_{0} Z^{8 / 9}} \cdot \frac{\left(1+0.978 \cdot 10^{-3} E_{0}\right)^{5 / 3}}{\left(1+1.957 \cdot 10^{-3} E_{0}\right)^{1 / 3}} .
$$

Here $A$ is the effective target material atomic weight, $\gamma=0.187 Z$ and $C \approx 1.1$.

Note that when modeling the diffusion process, the right-hand side of Eq. (2) should contain the concentration of the MCC $\rho(M)$ generated in the semiconductor, which is achieved by dividing $\rho *(M)$ from relation (3) by the energy of formation of an electronhole pair (or exciton). And for the two-dimensional model $z \approx z_{m s} \approx z_{s s}$.

The problem of assessing the correctness of mathematical models of the time-of-flight CL of direct-gap semiconductors was considered by us for two models describing the energy loss density of a primary electron beam: for a model using the classical Gaussian function $[1,3]$ and for a model that takes into account the features of energy losses in a condensed substance absorbed in the target and vice versa scattered electrons $[5,6]$. Using the standard approach [7] to assess the correctness of the considered mathematical models of diffusion (MCC or excitons) allowed us to obtain the following results:

1) following [13], for the two diffusion models under consideration, the existence and uniqueness of a solution to this problem is proved;

2) the continuous dependence of the solution of diffusion problems on the data of the problem is proved. The following estimates are obtained for the two models under consideration: if $n_{1}(M)$ is solution (2) with the right side $\rho_{1}(M)$, and $n_{2}(M)$ is solution (2) with the right side $\rho_{2}(M)$, and

$$
\left|\rho_{2}(M)-\rho_{1}(M)\right| \leq \varepsilon
$$

then:

for two-dimensional model $\left|n_{2}(M)-n_{1}(M)\right| \leq \lambda^{2} \varepsilon$,

for three-dimensional model $\left|n_{2}(M)-n_{1}(M)\right| \leq C \varepsilon, \quad C=\left(\lambda^{2}+\sqrt{26}\right) / 2$;

3) if $\left|n_{2}(M)-n_{1}(M)\right| \leq \delta$, for both two-dimensional and three-dimensional models

$$
\left|c_{2}(M, t)-c_{1}(M, t)\right| \leq \delta
$$

The dependence $I(t)$ was considered for low-energy beams (when the absorption of CL in the target volume can be neglected) [4]. In this case, in cylindrical coordinates

$$
I(t)=2 \pi \int_{0}^{R} c(r, t) r d r
$$


or

$$
I(t)=\frac{\pi \exp (-t / \tau)}{D t} \int_{0}^{R} \int_{0}^{+\infty} \exp \left(-\frac{r^{2}+\xi^{2}}{4 D t}\right) I_{0}\left(\frac{r \xi}{2 D t}\right) n(\xi) r \xi d \xi d r
$$

where $I_{0}(x)$ is the modified Bessel function of the first kind of the zero order. form

Using the Fubini theorem and the Poisson integral, we rewrite this expression in the

$$
\left|I_{2}(t)-I_{1}(t)\right| \leq \lambda^{2} \varepsilon \exp (-t / \tau) \cdot \iint_{H} d x d y
$$

and, therefore, the estimate

$$
\left|I_{2}(t)-I_{1}(t)\right| \leq \pi R^{2} \lambda^{2} \varepsilon \exp (-t / \tau) \leq \pi R^{2} \lambda^{2} \varepsilon
$$

is valid for $H=\{(t, x, y): t \geq 0, R>x \geq 0, R>y \geq 0\}$.

We note that the dependence of the CL intensity on time can be obtained in the polar coordinate system using the Hankel transform. Then for $c(r, t)$ we get

$$
c(r, t)=\frac{\exp (-t / \tau)}{2 D t} \int_{0}^{\infty} \exp \left(-\frac{r^{2}+\xi^{2}}{4 D t}\right) I_{0}\left(\frac{r \xi}{2 D t}\right) n(\xi) \xi d \xi .
$$

and for the dependence $I(t)$ we obtain the expression coinciding with (4).

\section{Simulation results}

Fig. 2 presents the results of mathematical modeling of the process of estimating the coefficient of diffusion of excitons by the least squares method. Modeling was carried out in the same way as in [10].

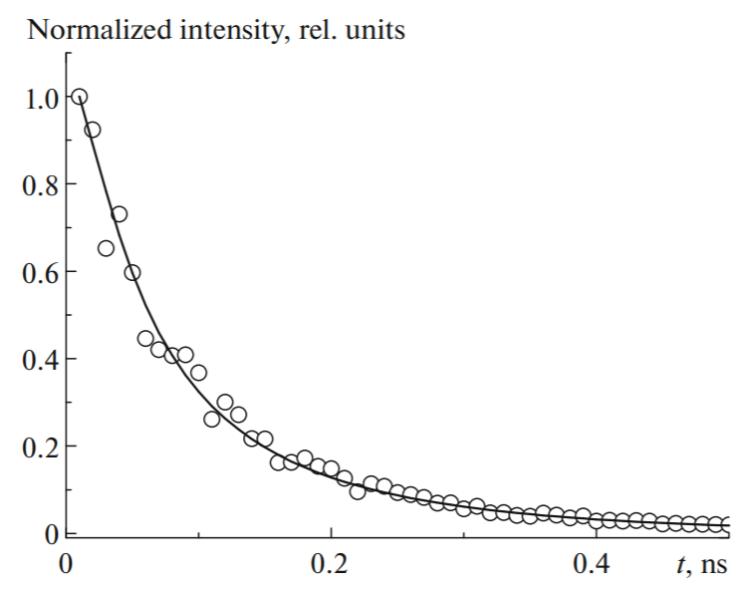

Fig. 2. Time dependence of normalized time-of-flight CL intensity for material parameters typical of $\mathrm{GaN}$ and a round aperture with $R=0.425 \mu \mathrm{m}$. Calculations were performed using formula (4) in the same way as in [10].

For the parameters of a GaN sample with $\tau=271 \mathrm{ps}$ and $D=5 \mathrm{~cm}^{2} / \mathrm{s}$ at $R=0.425 \mu \mathrm{m}$, the $\mathrm{CL}$ intensity was calculated as a function of time. The intensity values $I(t)$ calculated in this 
way were superimposed by random measurement errors in the form of normal random variables with zero mathematical expectation and a dispersion of $15 \%$ of the CL intensity amplitude at each point. The obtained points are indicated in Fig. 2 by white circles. Curve Fig. 2 corresponds to the CL intensity calculated for the optimal value $D=5.76 \mathrm{~cm}^{2} / \mathrm{s}$ obtained as a result of the application of the least squares method. Analysis of Fig. 2 shows a good agreement between the data obtained according to (4) with artificially introduced errors and the result of calculations using the least squares method, and the data obtained in [10] - they practically coincide at the selected scale.

Thus, expression (4) proposed for the description of exciton CL can be used to obtain estimates of the diffusion coefficient and exciton mobility by the method described above.

Moreover, an analysis of the results of mathematical modeling shows that during experimental time-of-flight studies of gallium nitride samples (exciton lifetimes of about 250-400 ps), correct results allowing an estimate of the exciton diffusion coefficient can be obtained by measuring in the center of the holes with a radius of $0.2-0.6 \mu \mathrm{m}$.

\section{Conclusions}

A comparative study of the qualitative properties of two-dimensional and three-dimensional mathematical models of diffusion and cathodoluminescence of excitons in single-crystal gallium nitride excited by a pulsating sharply focused electron beam in a homogeneous semiconductor material is carried out. For the mathematical model of the studied physical phenomenon, a study of its correctness has been carried out, estimates have been obtained to evaluate the effect of errors in the initial data on the distribution of the diffusing impurity and the cathodoluminescence intensity. The obtained results can be used in planning the experiment in electron-beam technologies.

This work was supported by the Russian Foundation for Basic Research, project no. 19-03-00271, and by the Russian Foundation for Basic Research and the Government of Kaluga District, project no. $18-41-400001$.

\section{References}

1. A.N. Polyakov, A.N. Smirnova, M.A. Stepovich, D.V. Turtin, Qualitative properties of a mathematical model of the diffusion of excitons generated by electron probe in a homogeneous semiconductor material, Lobachevskii Journal of Mathematics, v. 39, pp. 259-262 (2018)

2. M.A. Stepovich, D.V. Turtin, E.V. Seregina, and A.N. Polyakov, On the qualitative characteristics of a two-dimensional mathematical model of diffusion of minority charge carriers generated by a low-energy electron beam in a homogeneous semiconductor material, Journal of Physics: Conf. Series, v. 1203, 012095 (2019)

3. D.V. Turtin, E.V. Seregina, M.A. Stepovich, On the qualitative analysis of one class of differential equations of heat and mass transfer in condensed matter, Journal of Mathematical Sciences (to be published)

4. D.V. Turtin, M.A. Stepovich, E.V. Seregina, V.V. Kalmanovich, On the qualitative characteristics of a two-dimensional mathematical model of cathodoluminescence generated by a low-energy electron beam in a homogeneous semiconductor material, Journal of Mathematical Sciences (to be published)

5. N.N. Mikheev, V.I. Petrov, M.A. Stepovich, Quantitative analysis of semiconductor optoelectronic materials by raster electron microscopy, Bulletin of the Academy of Sciences of the USSR. Physical Series, v. 55, pp. 1-6 (1991) 
6. N.N. Mikheev, M.A. Stepovich, Distribution of energy losses in interaction of an electron probe with material, Industrial Laboratory, v. 62, pp. 221-226 (1996)

7. V.S. Vladimirov, V.V. Zharinov, Equations of Mathematical Physics: A Textbook for High Schools (Fizmatlit, Moscow, 2004)

8. M. Noltemeyer, F. Bertram, T. Hempel, B. Bastek, A.N. Polyakov, J. Christen, M. Brandt, M. Lorenz, M. Grundmann, Excitonic transport in $\mathrm{ZnO}$, Journal of Materials Research, v. 27, pp. 2225-2231 (2012)

9. A.N. Polyakov, M.A. Stepovich, D.V. Turtin, Three-Dimensional Diffusion of Excitons Generated by an Electron Beam in a Semiconductor Material: Results of Mathematical Modeling, Journal of Surface Investigation. X-ray, Synchrotron and Neutron Techniques, v. 9, pp. 1251-1255 (2015)

10. A.N. Polyakov, M.A. Stepovich, D.V. Turtin, Mathematical modeling of the cathodoluminescence of excitons generated by a narrow electron beam in a semiconductor material, Bulletin of the Russian Academy of Sciences: Physics, v. 80, pp. 1436-1440 (2016)

11. A.N. Polyakov, M. Noltemeyer, T. Hempel, J. Christen, M.A. Stepovich, Two Dimensional Diffusion and Cathodoluminescence of Excitons Generated by an Electron Beam in a Semiconductor Material: Results of Mathematical Modelling, Journal of Surface Investigation. X-ray, Synchrotron and Neutron Techniques, v. 6, pp. 901-905 (2012)

12. K Kanaya, and S. Okayama, Penetration and energy-loss theory of electrons in solid targets, J. Phys. D: Appl. Phys., v. 5, pp. 43-58 (1972)

13. A.N. Tikhonov, A.A. Samarskii, Equations of mathematical physics (Nauka, Main Edition of the Phys.-Math. Literature, Moscow, 1972) 